\title{
Propranolol treatment for infantile hemangioma: a case series of sixty-two patients
}

\author{
Giovanna Stringari,1 Giulia Barbato,1 Matteo Zanzucchi,1 Maddalena Marchesi,1 \\ Giuseppe Cerasoli,2 Bertrand Tchana,1 Alessandro Gritti,3 Valeria Boccaletti,4 \\ Alfonsa Anna Lombardi, 5 Nicola Carano 1
}

\section{Department of Pediatrics; 2Pediatric Surgery Unit; 3 Maxillofacial Surgery Unit; ${ }^{4}$ Department of Dermatology; 5Department of Radiology, Parma University Hospital, Parma, Italy}

\begin{abstract}
Infantile hemangiomas (IH) complicated by ulceration, disfigurement, functional impairment or life-threatening conditions need early, safe and effective treatment. This study explores the impact of propranolol on complicated $\mathrm{IH}$. We report our experience of 62 patients treated with oral propranolol for complicated IH. The effect of propranolol was assessed using a score on a visual analogue scale integrated with echo, magnetic resonance or endoscopic findings. The average age at the beginning of the treatment was seven months [standard deviation (SD) \pm 8.9 ], with a median of four months (range 1-53 months). The average age at the end of the treatment was 15 months $\left(\mathrm{SD}_{ \pm} 8.4\right)$, with a median of 13 months (range 7-59 months). The mean treatment length was eight months $(\mathrm{SD} \pm 3.2)$. Oral propranolol was successful in $95.2 \%$ of the patients in reducing the volume, the intensity of color and the elevation of IH. Statistically significant improvement of IH volume was observed in the first two months of therapy $(\mathrm{P} \leq 0.001)$, and between the second month and the end of the treatment $(\mathrm{P}<0.05)$. No significant bradycardia or hypotension occurred. Severe hypoglycemia occurred in
\end{abstract}

\footnotetext{
Correspondence: Nicola Carano, Department of Pediatrics, Parma University Hospital, via Bavagnoli 12, 43123 Parma, Italy.

Tel: +39.334.6896360.

E-mail: nicola.carano@gmail.com

Key words: Propranolol; Hemangioma; Infants.

Contributions: MM, MZ, GB and GS: data collection; GB, GS and NC: data analysis, data interpretation and writing of the manuscript; VB, AAL, NC, GC and AG: study design; BT: literature search and figures.

Conflict of interest: the authors declare no potential conflict of interest.

Note: approval for the study was obtained by the ethical committee of University of Parma for Medical Sciences.

Received for publication: 7 July 2015.

Revision received: 9 August 2015.

Accepted for publication: 1 June 2016.

This work is licensed under a Creative Commons Attribution NonCommercial 4.0 License (CC BY-NC 4.0).

(C) Copyright G. Stringari et al., 2016

Licensee PAGEPress, Italy

La Pediatria Medica e Chirurgica 2016; $38: 113$

doi:10.4081/pmc.2016.113
}

one patient. Mild adverse effects were observed in seven patients. Our study demonstrates that propranolol administered orally at 2 to 3 $\mathrm{mg} / \mathrm{kg} /$ day has a rapid therapeutic effect leading to remarkable shortening of the natural course of $\mathrm{IH}$ and it is safe in the majority of patients.

\section{Introduction}

Infantile hemangiomas (IH) are the most common tumors of infants, occurring in 1 to $4 \%$ of children under one year of age. ${ }^{1-3}$ They are composed of multipotent stem cells, immature endothelial cells, pericytes and mesenchimal cells. ${ }^{4}$ Infantile hemangiomas follow a typical course: they appear within the first few days to weeks after birth and usually grow rapidly during the first year of life, sometimes continuing up to 24 months. After a period of stabilization lasting a few months IH undergo spontaneous involution. This usually occurs after several years. 4

The pathogenesis of $\mathrm{IH}$ remains unknown. At present there are three main hypotheses: the theory of tissue hypoxia, the theory of embolization of placental endothelial cells, and the theory of increased angiogenic and vasculogenic activity. 5,6 The possibility that the three mechanisms could act together is not excluded. ${ }^{6}$ Whatever the pathogenic mechanism, in the proliferative phase of IH pro-angiogenic factors, vascular endothelial growth factor (VEGF), and basic fibroblast growth factor (bFGF) show increased expression, whereas during the regressive phase the levels of VEGF and bFGF are significantly lower.5,6

The vast majority of IH do not have a clinical impact and do not need any treatment. However, in some patients IH significantly progress and can be complicated by ulceration; they may lead to permanent scars or disfigurement; or they may cause functional impairment (visual or auditory) or life-threatening conditions (airway obstruction or cardiac compromise). In these cases prompt therapy should be considered to prevent or treat the complications. In 2008 Leaute-Labreze noted the positive effect of propranolol on a nasal IH. ${ }^{7}$ Since then other studies have confirmed the efficacy of oral propranolol therapy, which has become the first-line treatment for IH. - $-14^{-14}$

In December 2009 at the Department of Pediatrics of Parma University an observational study on oral propranolol therapy for IH was started. The aim of this clinical trial was to evaluate the efficacy and safety of propranolol treatment for IH and to verify the appropriateness of our diagnostic-therapeutic approach.

\section{Materials and Methods}

From December 2009 to August 2014 we enrolled 68 infants in whom the treatment with propranolol was indicated in order to prevent 
or ameliorate a complication by an IH. The first referral and the indication for treatment with propranolol was decided on the basis of the guidelines of the American Academy of Dermatology 15 by a medical team consisting of a pediatrician, a dermatologist, a pediatric surgeon, a plastic surgeon and a pediatric radiologist. In four patients informed consent was not achieved, so the treatment was started in 64 patients. Two patients were subsequently excluded from the study due to poor compliance with the treatment.

A total of 62 patients were treated. Parents gave their written consent to start the treatment with propranolol and to acquire serial photos in order to evaluate the initial appearance of the lesion, its growth and its evolution during the treatment. All infants were hospitalized in our pediatric ward for 48 hours in order to start the treatment under medical control. A careful patient history was recorded and a physical examination was performed to assess risk factors or contraindications to using propranolol, such as reactive airway disease, asthma, cardiac disease, cardiac arrhythmia, hypoglycemia. If these last contraindications were present, additional work-up was required. All patients underwent a baseline electrocardiogram that was interpreted by a pediatric cardiologist. If abnormal cardiac findings at history or physical examination or electrocardiography (ECG) abnormalities were found, echocardiography was performed. In case of deep IH, echography was performed to measure the extent or to determine the nature of the lesion. When we were not able to define the nature or the extent of the lesions by clinical findings or echography, magnetic resonance imaging (MRI) was performed.

During the hospital stay the patient was evaluated clinically twice a day by a pediatrician. The heart rate and blood pressure were measured before the administration of propranolol and 2 hours afterwards to coincide with plasmatic peak level. Propranolol was started at a dose of 1 $\mathrm{mg} / \mathrm{kg} /$ day in three divided doses for the first 24 hours, then it was increased to $2 \mathrm{mg} / \mathrm{kg} /$ day. The parents were trained to manage the treatment at home and to recognize early adverse events and conditions that could require interruption of the treatment (vomiting, diarrhea, reduction in feeding, wheezing, respiratory distress, irritability/apathy).

Four to seven days after discharge from hospital, in the absence of adverse reactions, the dosage was increased to $2.5-3 \mathrm{mg} / \mathrm{kg} /$ day in three divided doses. Monthly clinical evaluation was performed, with measurement of heart rate and blood pressure; acquisition of new pictures of the lesion; adjustment of the dosage of the drug; and, if necessary, echography. In all patients the treatment was started using an oral solution containing $1 \mathrm{mg} / \mathrm{mL}$ of propranolol; the therapy was continued with a powder galenical formula in some cases and in other cases with an oral solution containing $2 \mathrm{mg} / \mathrm{mL}$ of propranolol, depending on the pharmacy supplying the drug.

The results of the therapy were evaluated through the visual analogue scale (VAS) proposed by Zvulunov.16 The VAS scale is based on the evaluation, made by two independent observers, of photographic documentation acquired before, during and after the treatment. The scale consists of values between zero and ten, where ten indicates the worst aspect of IH and zero that IH is no longer visible. A score of five means an IH decrease of $50 \%$ compared with its worst aspect. In the case of mixed, or exclusively deep IH, or in those IH invisible from the outside the VAS score was assigned on the basis of ultrasound, MRI and endoscopic findings, as well as on the appearance of the affected area in the photographs.

The end of the therapy was established considering the age of the patient (the IH had to be close to the end of the growing phase) and the results obtained in terms of reduction in volume and stability of the lesion. Once the decision to suspend the treatment had been taken, the dosage was gradually tapered and the drug was discontinued after two weeks. Follow-up visits were made at one and six months after the end of the therapy.

The results during therapy and six months after the cessation of treatment were analyzed with SPSS Statistics 19.

\section{Results}

Sixty-two patients suffering from IH were treated with oral propranolol. The clinical indication for the treatment was: disfigurement in 41, ulceration in five and functional impairment in 16 (visual in 11, auditory in three, airway in two).

Forty-two patients were females and twenty were males ( $\mathrm{F} / \mathrm{M}=2.1: 1)$. Eleven patients (17.7\%) were ex-preterm neonates ranging from 30 to 37 weeks of gestational age. Three patients were born of a twin pregnancy (the other twin was free of $\mathrm{IH}$ ). The average age at the appearance of $\mathrm{IH}$ was 14.5 days $(\mathrm{SD} \pm 20.8)$, the median was seven days (range 0-90 days). We treated 53 single lesions and 11 multiple lesions (the total number of lesions was 71 in 62 patients). Considering the anatomical location of the IH, 47 (66.2\%) were located on the head, 13 (18.3\%) on the torso, 7 (9.9\%) on the limbs, and 4 (5.6\%) on the neck. In only one patient all of the lesions (four superficial IH) were significant enough to require treatment. There were 65 lesions requiring treatment in 62 patients: 20 (30.8\%) were superficial, 13 (20\%) were deep, and $32(49.2 \%)$ were mixed. One of the deep IH involved the parotid gland (Figure 1). The IH treated was in proliferative phase in
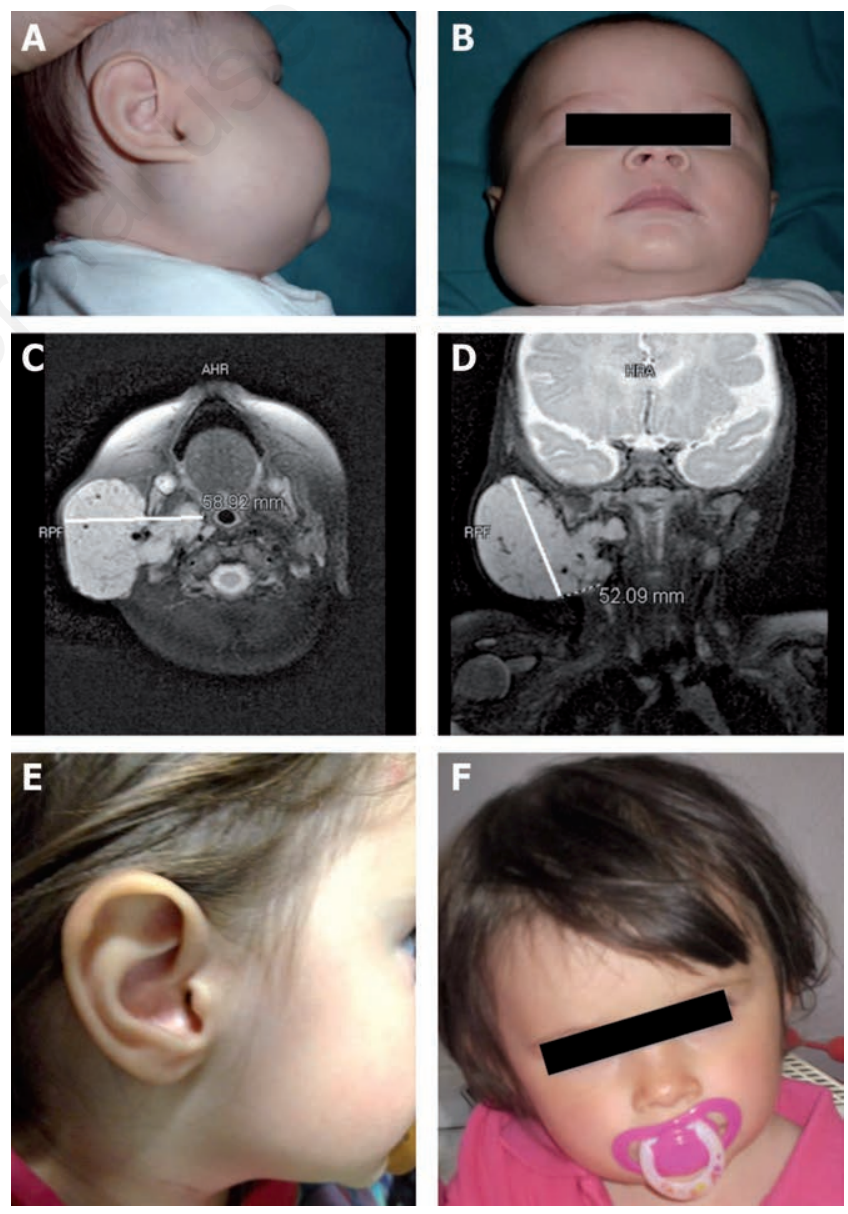

Figure 1. A,B) Appearance of an infantile hemangioma (IH) involving the parotid gland in a 5 month-old infant. C,D) In magnetic resonance imaging the lesion occupies the parotid region extending up to parapharyngeal space. The parotid parenchyma is not visible. E,F) Patient's appearance at 23 months of age at the time of cessation of the treatment. The large parotid IH is completely regressed. 
57 (92\%) patients, in 5 (8\%) patients the lesion was stable when our team saw it for the first time, but there was significant esthetic impairment that justified the beginning of therapy (Table 1).

Two patients suffered from IH in the setting of a more complex condition: one had PELVIS syndrome (perineal IH, external genital malformations, lipomyelomeningocele, urinary tract abnormalities, imperforate anus and skin tag) and one had SACRAL syndrome (spinal dysraphism, anogenital, cutaneous, renal and urologic anomalies, associated with a lumbosacral $\mathrm{IH}$ ).

Echography of the IH was performed in 25 patients, and MRI in four. Echocardiography was performed in one patient with abnormal cardiac findings and ECG abnormalities. Multiple atrial septal defects were found. As is typical in children with atrial septal defects, no clinical signs of heart failure were present in this patient.

The average age at the beginning of treatment was seven months ( $\mathrm{SD} \pm 8.9$ ), with a median of four months (range 1-53 months). The average age at the end of treatment was 15 months $(\mathrm{SD} \pm 8.4)$, with a median of 13 months (range 5-59 months). The mean treatment length was eight months (range 1-21 months, $\mathrm{SD} \pm 3.2$ ). In order to evaluate the results of the treatment the VAS scores were analysed with Wilcoxon's test. The median of the VAS scores was: seven (range 5-10), six (range 2-10), four (range 1-10), three (range 1-8), and three (range 1-10), respectively at the first, second, third, and sixth month and at the end of the treatment. There was a significant statistical improvement of VAS scores, in particular between the beginning and the first month of the therapy $(\mathrm{P}<0.001)$, between the first and the second month $(\mathrm{P}=0.001)$, between the second and the sixth month $(\mathrm{P}<0.05)$ and between the second and the end of treatment $(\mathrm{P}<0.05)$ (Figure 2 and Table 2$)$.

In seven patients (11.3\%) the residual IH began to grow significantly again after discontinuation of the therapy (6/7 of relapsing IH had a deep component and $2 / 7$ were segmental). A second cycle of propranolol was administered with the same beneficial result as the first treatment and without any relapse. The average age at the beginning of the second treatment was 13.1 months $(\mathrm{SD} \pm 2.9)$, with a median of 12.5 months (range 9-19 months). The average length of the second cycle of treatment was 6.25 months $(\mathrm{SD} \pm 1.8)$. Considering the morphology of the IH requiring a second cycle of treatment, one (14\%) was superficial, three (43\%) were deep and three (43\%) were mixed, the anatomical location was in $5(72 \%)$ cases the head, in 1 (14\%) case the torso and in $1(14 \%)$ case the neck.

The patients who responded to the treatment with oral propranolol were 59/62 (95.2\%). The effect appeared quickly in the first few days

Table 1. Characteristics of the population sample.

\begin{tabular}{lcc} 
& \multicolumn{2}{c}{ All (n=62) } \\
Females (n, \%) & 42 & 68 \\
Preterms (n, \%) & 11 & 18 \\
\hline Twins (n, \%) & 3 & 5 \\
Age at appearance of IH (mean, SD) & 14.5 & 20.8 \\
\hline Multiple IH (n, \%) & 11 & 18 \\
Location of IH (n, \%) & 71 & \\
Head & 47 & 66 \\
Torso & 13 & 18 \\
Limbs & 7 & 10 \\
Neck & 4 & 6 \\
\hline Lesion treated (n, \%) & 65 & \\
Superficial & 20 & 31 \\
Deep & 13 & 20 \\
Mixed & 32 & 49 \\
\hline
\end{tabular}

IH, infantile hemangioma; SD, standard deviation. Age of patients is expressed in days. and was sustained during the following weeks leading to a remarkable shortening of the natural course of IH. Furthermore, the infants who started therapy during the first six months of age showed a greater improvement of the lesions. The average VAS score after the first month of therapy was similar in the two groups, seven (range 5-10) for the infants that started treatment before six months of age and eight (range 5-10) for those that started treatment later. By contrast, at the end of therapy the average VAS score was two (range 1-10) for the infants that began propranolol before six months of age and six (range $1-10)$ for the others $(\mathrm{P}<0.05)$. Figure 3 shows the effect of treatment on a superficial IH.

Three patients (4.8\%) did not respond to the treatment. In two the VAS score at the end of the therapy was ten and nine. In both cases the treatment had been started after one year of age. They underwent elective surgery. The other patient, whose VAS score after one month of treatment was ten, required urgent surgery because of massive bleeding (Table 3).

None of the 62 patients presented bradycardia ( $<2 \mathrm{SD}$ of normal), or hypotension ( $<2 \mathrm{SD}$ of normal). One of our first patients, a 53 monthold girl who had already unsuccessfully been treated with oral pred-

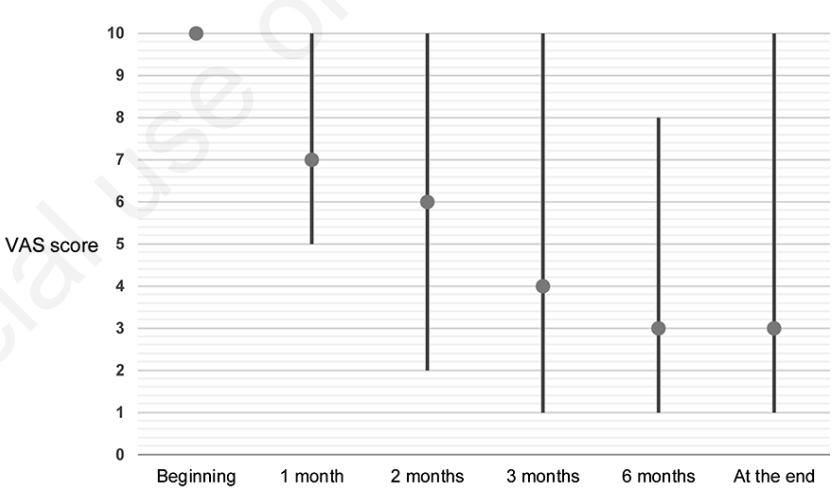

Figure 2. Infantile hemangioma visual analogue scale median scores at the beginning, first, second, third, sixth month and at the end of treatment with propranolol.

Table 2. Analysis of treatment.

\begin{tabular}{lcc} 
& \multicolumn{2}{c}{ All (n=62) } \\
Age at the beginning (mean, SD) & 7 & 8.9 \\
Age at the end (mean, SD) & 15 & 8.4 \\
\hline Length (mean, SD) & 8 & 3.2 \\
VAS score (median, range) & 7 & \\
1 month & 6 & $5-10$ \\
2 months & 4 & $2-10$ \\
3 months & 3 & $1-10$ \\
6 months & 3 & $1-8$ \\
At the end & 7 & $1-10$ \\
\hline Recurrence (n, \%) & 13.1 & 11 \\
Age at recurrence (mean, SD) & 6.25 & 2.9 \\
Length (mean, SD) & 8 & 1.8 \\
Side effects (n, \%) & 6 & 13 \\
Sleep disorders & 1 & 10 \\
Hypoglycemia-induced seizure & 1 & 2 \\
Transient alopecia &
\end{tabular}

SD, standard deviation; VAS, visual analogue scale. Age of patients is expressed in months. 
nisone, started therapy with propranolol and after 6 months of treatment (VAS from ten to eight) suffered from a hypoglycaemia-induced seizure during an episode of gastroenteritis. Six infants (9.6\%) had sleep disorders requiring a slight reduction of the propranolol dosage. One patient (1.6\%) presented transient alopecia. Eight patients (12.9\%) had to stop the therapy briefly because of wheezing or bronchiolitis. In two patients (3.2\%), because of gastroenteritis and poor feeding, a short interruption of the therapy was necessary as a precaution in order to decrease the risk of hypoglycemia.

In order to obtain a better cosmetic result, five patients, who had successfully reached the end of the treatment, underwent elective surgery to remove residual scars.
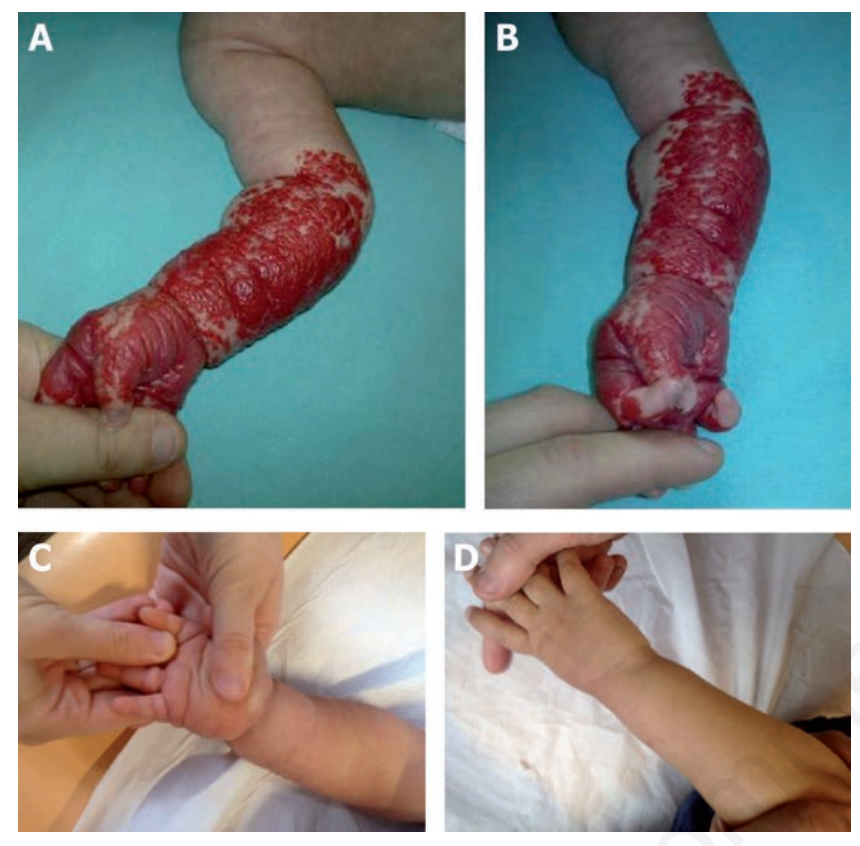

Figure 3. A,B) Appearance of superficial infantile hemangioma of the arm and hand at 2 months of age; C,D) complete regression of the lesion after 10 months of treatment with propranolol.

Table 3. Characteristics of non-responders.

\begin{tabular}{lcc} 
& \multicolumn{1}{c}{ AlI (n=3/62) } \\
Females (n, \%) & 2 & 67 \\
Preterms (n, \%) & 0 & 0 \\
\hline Twins (n, \%) & 0 & 0 \\
Age at appearance of IH (mean, SD) & 11 & 15.5 \\
\hline Multiple IH (n, \%) & 1 & 33 \\
Location of IH (n, \%) & 4 & 25 \\
Head & 1 & 50 \\
Torso & 2 & 0 \\
Limbs & 0 & 25 \\
Neck & 1 & \\
\hline Lesion treated (n, \%) & 4 & 25 \\
Superficial & 1 & 25 \\
Deep & 1 & 50 \\
Mixed & 2 & \\
\hline
\end{tabular}

IH, infantile hemangioma; SD, standard deviation. Age of patients is expressed in days.

\section{Discussion}

The majority of IH have little impact on infants' and children's health, but a few of them, especially those that are located on the head, face and neck and develop rapidly, can interfere with normal function and appearance. In these cases a prompt intervention is required in order to control growth and reduce the probability of functional and cosmetic disorders.

Propranolol is a nonselective $\beta$-adrenergic receptor blocker commonly used in the pediatric age group for the treatment of supraventricular and ventricular tachyarrhythmias; obstructive hypertrophic cardiomyopathy; hypertension; treatment and prophylaxis of anoxic spells in tetralogy of Fallot; prophylaxis of migraine; and as an adjuvant drug in the treatment of thyrotoxicosis, pheochromocytoma and portal hypertension with esophageal varices. Rare side effects of propranolol are bradycardia, atrio-ventricular block, hypotension, bronchospasm, hyperkalemia. Common side effects are: sleep disorders, alopecia, feeding and intestinal disorders. ${ }^{17}$ The use of propranolol in infants and children was off-label in Italy, but in 2010 the Italian Drug Agency (Agenzia Italiana del Farmaco; AIFA) authorized its use in infants with severe $\mathrm{IH}$.

The effect of propranolol on growing IH can be ascribed to three molecular mechanisms: vasoconstriction, inhibition of angiogenesis and induction of apoptosis. The vasoconstriction occurs because propranolol inhibits the vasodilator effect of adrenaline via beta-receptors, but does not affect the activity of adrenalin on alfal-receptors. ${ }^{18,19}$ The inhibition of angiogenesis occurs because of the effects of adrenaline and noradrenaline, which consist in the stimulation of the synthesis of the pro-angiogenic factors VEGF and bFGF, which in turn are inhibited by the propranolol-induced beta-block. ${ }^{19}$ Another mechanism to explain the effect of propranolol in accelerating the regression of proliferating infantile emangioma is linked to its action on the renin-angiotensin system, as proposed by Itinteang and co-authors. ${ }^{20}$ They demonstrated that CD34+/CD33+/NEGFR+ cells present within the immature capillaries of proliferating $\mathrm{IH}$ are responsive to angiotensin II and suggested that beta-blockers, acting on the kidney, reduce renin activity and consequently the agiotensin II, a factor that stimulates mesenchimal stem cells of IH to secrete the VEGF. Regarding apoptosis, adrenergic stimulation inhibits apoptosis through the intervention of several enzymes (src, mitogen-activated protein kinases, caspase cascade), thus the adrenergic block induced by propranolol can increase the cellular apoptosis rate. ${ }^{18,19}$

Since the initial report of propranolol use for the treatment of IH, some published case series $8-14$ and many case reports have confirmed its effectiveness in reducing the sizes of IH in 82 to $100 \%$ of treated patients and have proved the safety of the treatment. The report of a consensus conference on the initiation and the use of propranolol for IH was published in 2013.17 In 2015 a randomized controlled trial showed that propranolol was effective at a dose of $3 \mathrm{mg} / \mathrm{kg}$ per day for six months in the treatment of IH. ${ }^{21}$

In our study the efficacy of propranolol reached $95.2 \%$. In responders the first effects of propranolol appeared during the first 24 hours of treatment as changes in color (from bright red to purple) and consistency (softening of the lesion on palpation). After the initial response IH continued to progressively improve both with respect to color and thickness. The highest statistical significance of clinical improvement occurred in the first two months of treatment $(\mathrm{P} \leq 0.001)$, but IH continued to improve in the following months $(\mathrm{P}<0.05)$. Patients who started treatment before six months of age showed the best results in terms of the VAS score at the end of treatment. Therefore, it appears that early recognition and early treatment of $\mathrm{IH}$ assure the best outcome. Ulcerations healed in a few days in all patients except in the one who required urgent surgery because of massive bleeding. Clinically signif- 
icant regrowth of IH was observed in 7/62 patients (11.3\%) and was treated with a second propranolol therapy regimen. The rate of relapse in our study was similar to that found by Ahogo and colleagues, who found $12 \%$ of patients having a major relapse with a true regrowth phase of the IH necessitating a new oral course of propranolol. They also found that children at risk of relapse were mostly those with segmental IH, and those with IH with a deep component. ${ }^{22}$ This was very similar to what we found in our series. The percentage of IH that did not respond to the treatment was $4.8 \%$ in our series. This value is greater than the value of treatment failures estimated in the consensus conference about propranolol use for IH (1.6\%), however the authors themselves pointed out that this failure rate could be underestimated because treatment failures were not so commonly reported (only 14\% of the publications examined addressed the issue of treatment failures). ${ }^{17}$

In our study the first assessment of the patients referred for IH was made by a medical team. We think that this strategy, summing the experience of many specialists, greatly reduces the possibility of a diagnostic error and ensures the selection of the most appropriate therapy.

Our policy was to start the treatment during a 48-hour hospital stay in order to monitor the tolerance to the therapy and the outbreak of adverse effects. All our patients were discharged from the hospital after 48 hours with a propranolol dosage of $2 \mathrm{mg} / \mathrm{kg} /$ day in three divided doses. We did not observe any side effects in the first 48 hours. Therefore, our method to start propranolol therapy at the dosage of 1 $\mathrm{mg} / \mathrm{kg} /$ day and to increase it up to $2 \mathrm{mg} / \mathrm{kg} /$ day after 24 hours was free of side effects and can be considered safe. This outcome convinced us to start the treatment as outpatients in subsequent cases using a fourhour period of observation beginning at the first dose of propranolol. This seems to be adequate to recognize bradycardia or hypotension because the propranolol plasmatic peak level occurs after two hours. Hospitalization should be reserved for patients with simultaneous occurrence of premature birth or other conditions affecting the cardiovascular system, the respiratory system or blood glucose maintenance, wherein propranolol is more likely to cause adverse effects.

In our series adverse effects were observed in eight patients (12.9\%). This value is lower than the overall value of adverse effect estimated in the consensus conference (15.5\%). ${ }^{17}$ Seven of the eight showed mild adverse effects and only one patient (1.6\%) suffered from a severe adverse effect of propranolol (a hypoglycemia-induced seizure occurring during the course of gastroenteritis). Hypoglycemia in the setting of IH treated with propranolol has previously been reported. ${ }^{23-25}$ As the use of propranolol in infants with IH increases, physicians must be aware of the potential for hypoglycemia. It can be particularly insidious and difficult to recognize because some symptoms that are associated with counter-regulatory epinephrine action such as sweating, shakiness, anxiety, hunger and tachycardia may be absent, due to propranolol-induced $\beta$-adrenergic blockage. Therefore, in order to decrease the risk of hypoglycemia, propranolol should be discontinued during intercurrent illness, especially in the setting of restricted oral intake. Furthermore, patients with IH may be at increased risk of hypoglycemia if they have recently received or are concomitantly receiving corticosteroids, because adrenal suppression may lead to a failure of counter-regulatory cortisol response. ${ }^{2} 6$

\section{Conclusions}

In conclusion, in our study propranolol administered orally at 2 to 3 $\mathrm{mg} / \mathrm{kg} / \mathrm{day}$ in three divided doses was effective in $95.2 \%$ of cases. The effect appeared quickly in the first few days and was sustained during the following weeks leading to a remarkable shortening of the natural course of IH. The treatment failure rate was $4.8 \%$. Propranolol was well tolerated with only one (1.6\%) severe adverse effect. Starting the treatment before six months of age led to better results. Regrowth of the lesion benefited from a second treatment.

\section{References}

1. Frieden IJ, Haggstrom A, Drolet BA et al. Infantile hemangiomas: current knowledge, future directions: proceeding of a research workshop on infantile hemangiomas. Pediatr Dermatol 2005;22: 383-406.

2. Dickison P, Christou E, Wargon 0. A prospective study of infantile hemangiomas with a focus on incidence and risk factors. Pediatr Dermatol 2011;28:663-9.

3. Haggstrom AN, Drolet BA, Baselga E, et al. Prospective study of infantile hemangiomas: clinical characteristics predicting complications and treatment. Pediatrics 2006;118:882-7.

4. Chang LC, Haggstrom AN, Drolet BA, et al. Growth characteristics of infantile hemangiomas: implications for managements. Pediatrics 2008;122:360-7.

5. Bauland CG, van Stensel MA, Steijlen PM, et al. The pathogenesis of infantile hemangiomas: a review. Plast Reconstr Surg 2006;117:29-35.

6. Janmohamed SR, Madern GC, de Laat PCJ, et al. Educational paper: pathogenesis of infantile hemangioma, an update 2014 (part I). Eur J Pediatr 2015;174:97-103.

7. Leaute-Labreze C, Dumas de la Roque E, Hubiche T, et al. Propranolol for severe hemangionas of infancy. New Engl J Med 2008;358:2649-51.

8. Sans V, Dumas de la Roque E, Berge J, et al. Propranolol for severe infantile hemangiomas: follow-up report. Pediatrics 2009; 124:423-31.

9. Hogeling M, Adans S, Wargon 0. A randomized controlled trial of propranolol for infantile hemangiomas. Pediatrics 2011;128: 259-66.

10. Manunza F, Syed S, Laguda B, et al. Propranolol for complicated infantile hemangiomas: a case series of 30 infants. Brit J Dermatol 2010;162:466-8.

11. Schiestl C, Neuhaus K, Zoller S, et al. Efficacy and safety of propranolol as first-line treatment for infantile hemangiomas. Eur $\mathrm{J}$ Pediatr 2011;170:493-501.

12. Buckmiller LA, Munson PD, Dyamenahalli U, et al. Propranolol for infantile hemangiomas: early experience at a tertiary vascular anomalies center. Laryngoscope 2010;120:676-81.

13. Bagazgoitia L, Torrelo A, Gutierrez JC, et al. Propranolol for infantile hemangiomas. Pediatr Dermatol 2011;28:108-14.

14. Schneider M, Cremer HJ, Ruef P. A retrospective analysis of systemic propranolol for the treatment of complicated infantile hemangiomas. Acta Paediatr 2014;103:977-83.

15. Frieden IJ, Eichenfield LF, Esterly NB, et al. Guidelines of care for hemangiomas of infancy. J Am Acad Dermatol 1997;37:631-7.

16. Zvulunov A, McCuaig C, Frieden IJ, et al. Oral propranolol therapy for infantile hemangiomas beyond the proliferation phase: a multicenter retrospective study. Pediatr Dermatol 2011;28:94-8.

17. Drolet BA, Frommelt PC, Chamlin SL, et al. Initiation and use of propranolol for infantile hemangioma: report of a consensus conference. Pediatrics 2013;131:128-40.

18. Storch $\mathrm{CH}$, Hoeger PH. Propranolol for infantile hemangiomas: insights into the molecular mechanism of action. Brit J Dermatol 2010;163:269-74.

19. Chim H, Armijo BS, Miller E, et al. Propranolol induces regression of hemangioma cells through Hif-1 -mediated inhibition of VEGFA. Ann Surg 2012;256:146-56.

20. Itinteang T, Brasch HD, Tan ST, et al. Expression of components of 
the rennin-angiotensin system in proliferating infantile hemangioma may account for the propranolol-induced accelerated involution. J Plast Reconstr Aesthet Surg 2011;64:759-65.

21. Leaute-Labreze C, Hoeger P, Mazereeuw-Hautier J, et al. A randomized, controlled trial of oral propranolol in infantile hemangioma. New Engl J Med 2015;372:735-46.

22. Ahogo CK, Ezzedine K, Prev S, et al. Factors associated with the relapse of infantile hemangiomas in children treated with oral propranolol. Brit J Dermatol 2013;169:1252-6.

23. De Graaf M, Breur JM, Raphael MF, et al. Adverse effects of propra- nolol when used in the treatment of hemangiomas: a case series of 28 infants. J Am Acad Dermatol 2011;65:320-7.

24. Bonifazi E, Acquafredda A, Milano A, et al. Severe hypoglycemia during successful treatment of diffuse hemangiomatosis with propranolol. Pediatr Dermatol 2010:27:195-6.

25. Holland KE, Frieden IJ, Frommelt PC, et al. Hypoglycemia in children taking propranolol for the treatment of infantile hemangioma. Arch Dermatol 2010;146:775-8.

26. Breur J, de Graaf M, Breugem CC, et al. Hypoglycemia as a result of propranolol during treatment of infantile hemangioma: a case report. Pediatr Dermatol 2011;28:169-71. 